\section{Pendulums in practice}

\author{
Paul Foulkes
}

My Own Right Time: An Exploration of Clockwork Design. By Philip Woodward. Oxford University Press: 1995. Pp. 176. £24.99.

To set out the general theory of the ideal pendulum is a fairly simple exercise in mathematics. The complications that arise when we examine how the device works in practice are altogether more intricate. Philip Woodward's book gives a splendid

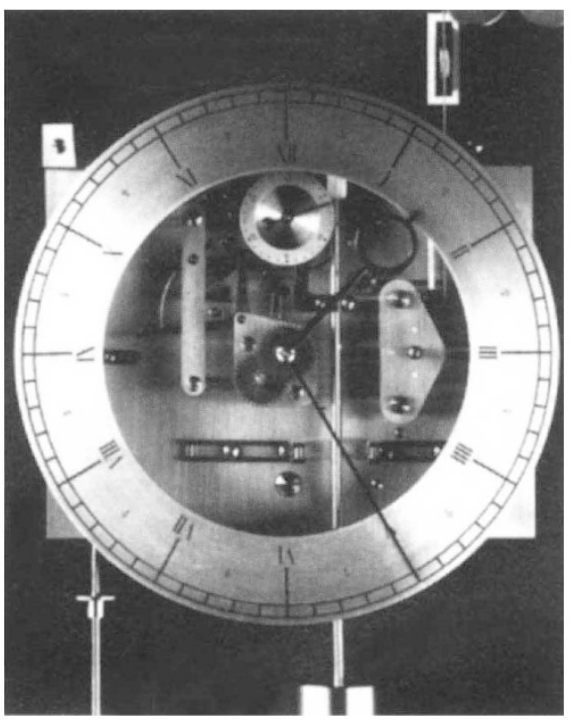

Clockwise - Woodward's remarkably accurate free pendulum clock W5.

account of this, with photos, drawings and graphs. While relating his own quest for an ever-more effective design for a mechanical time-keeper (his W5), he manages in clear and simple words to describe the main types of escapement mechanisms in clockwork right to the latest developments in mechanical art. For the purpose of keeping time, this has now been overtaken by the electronic revolution. Even so, much in mechanical horology remains both valuable and instructive in the field of applied mechanics.

The explanations of how these various devices work is supported by clear diagrams. To follow operations step by step is not always easy, but then the sequences are fairly complex, so that the reader must concentrate. The amount of mathematics is small and elementary, and calculus is avoided. This must have meant some selfrestraint, since the author is an applied mathematician and physicist. Still, the effort is commendable, in view of the wide readership envisaged. Perhaps in a later edition the meagre appendix could carry some more theory. Meanwhile, we have here a study of great depth of the problems of how to overcome the generally nonconservative damping forces we meet in practice.

To describe the quality of resonance in the resonator that constitutes the regulating mechanism, the symbol $Q$ has been borrowed from electric circuit theory. This is the ratio of peak restoring force to peak damping force, a dimensionless number. The greater $Q$, the more efficient the resonator and therefore the more accurate the time-keeping. Woodward tells us that "for some reason horologists did not at first take kindly to it". The reason is surely clear: the inverse ratio, peak damping to peak restoring force, is intuitively more intelligible. The smaller that ratio, the better and more efficient the resonator. To achieve this, we must reduce the damping force, rather than indefinitely increase the restoring force. Hence $Q^{\prime}=1 / Q$ is a quantity more easily visualized. To the mathematician this hardly matters, but it does to the practical mechanic.

In trying to give an intuitive account of the pendulum's basic lack of isochronism, the author bids us to imagine a pendulum swinging half a turn and coming to rest upside down. This is not a happy sug- gestion, because such a position of 'rest' is unstable; the pendulum would in fact topple over. The 18 per cent rise in period for a swing of 90 degrees is computable from the general solution of the pendulum equation, which involves an elliptic integral and is therefore rightly omitted.

The chapters on statistical error analysis and on flicker noise take the subject rather further than most ordinary accounts. Finally, there is some discussion of a source of error against which no mechanical remedy exists. This is the possible seasonal variation of the acceleration due to gravity at the Earth's surface, $g$. This can occur when the density in a layer of material below ground changes, for example when water is absorbed by porous rock in the rainy season.

Such changes are not precisely predictable: the user simply has to adjust his clock in the event. Changes in a pendulum's length, thermal disturbances and frictional losses in various mechanisms can be allowed for, but changes in natural 'constants' are much more elusive.

Paul Foulkes is at 24 Granville Park, London SE13 TEA, UK.

\title{
Evolution of artificial intelligence
}

\section{Terrence J. Sejnowski}

Artificial Minds. By Stan Franklin. MIT Press: 1995 . Pp. 449. \$30, £22.95.

WE are living in a postmodern age in which the functionalist architecture of artificial intelligence (AI), like the functional architecture of the Bauhaus, is beginning to look rather old fashioned. Stan Franklin takes the reader on a tour of the eclectic computational styles competing to replace the traditional symbol-processing paradigm that served as the unifying framework, and strait-jacket, for a generation of mindmodellers. He leads the mindful tourist on a whistle-stop tour of artificial neural networks, computational neuroethology, situated action, artificial life, chaos theory, quantum computing, neural Darwinism and a host of other hopeful approaches. Where symbolic AI was once the only game in town, there now seems to be one on every street corner.

A trend that has evolved over the past decade is a shift away from the toy worlds of early AI towards problems that arise from interaction with the real world. The first computers available to AI researchers had less computing power than a pocket calculator. So the problems attacked were highly simplified. Because computers are efficient at symbol processing, solutions were sought in the realm of logic. The world, however, is noisy and uncertain and does not come labelled by symbols, which must be grounded in sensation and learned through experience. Inductive reasoning from incomplete knowledge is as important as deductive reasoning. Ironically, it is not logic but the theory of gaming that provides the mathematical framework for inductive reasoning in an uncertain world, and George Boole, in his 1854 book An Investigation into the Laws of Thought, which introduced Boolean logic, devoted the second half to the theory of probabilities.

Artificial Minds is organized around three debates in AI. The first is whether a computer can ever be programmed to think. This is a recurring question that does not seem to have a resolution, in part because the definition of thinking keeps changing. The problem has been revived recently by Roger Penrose, who doubts the possibility that algorithms could ever think like a mathematician.

I am highly sceptical of claims that something is impossible. A small change in an innocent-looking assumption can drastically change the conclusions. Some aspects of thinking have already been automated, but there are others, such as subjective feelings, that do not have an obvious mechanical analogue. There is a vast middle ground to explore and much of it can be approached with empirical studies of brains and the invention of ever-more clever devices.

The second AI debate, led by the con- 
nectionist alternative to symbol processing, concerns Jerome Feldman's 100-step rule. The brain can solve many difficult cognitive problems in half a second. If a neuron takes 5 milliseconds to perform one operation, then it should be possible to devise algorithms for solving the same problems that require only 100 steps. Although the SOAR program of the late Allen Newell respected this constraint, most AI programs take millions of steps; connectionist algorithms, by contrast, distribute the information over many simple processing units and work in parallel. The first-generation neural network models, however, were weakest where symbol processing is strongest, in representing relationships between abstract categories. The analysis of nonlinear networks as dynamical systems is still in its infancy and I suspect that the next generation of network models, based on spiking neurons, may provide additional computational abilities that may overcome this limitation.

The third debate reaches into the heart of AI and questions the need for representations at all. Rodney Brooks incorporates a hierarchy of reactive behaviours into walking machines that inhabit the Massachusetts Institute of Technology; the emphasis is on action selection rather than highly detailed sensory representations. Of course, as the control of actions becomes more sophisticated, the burden of intelligence is simply shifted from the sensory to the motor side. Franklin declares his own bias for a theory of intelligence based on an action-selection scheme, in which tasks are solved by a variety of specialized agents. These autonomous agents remind me of programs on the World Wide Web, such as 'spider' agents that seek out and catalogue information available at Web sites. If the digital computer served as a model of the mind for a generation of students in cognitive science, will the next generation be guided by their experience surfing on the Internet?

Artificial Minds is a readable guide to the evolution of ideas in artificial intelligence. It is intended for a general audience that already has some familiarity with symbolic AI and could be used, like a tour guide, to decide what areas of nouvelle AI to visit in greater depth. My only disappointment is that cognitive neuroscience, the science of real minds, plays only a small part in the book, perhaps because this field is of less interest to the author, whose background is in mathematics and computer science. New techniques for probing brains in action are uncovering unexpected insights into the organization of human minds. But that is another tour.

Terrence J. Sejnowski is at the Salk Institute, 10010 North Torrey Pines Road, La Jolla, California 92037, USA.

\section{Hard to swallow}

\section{Bruce Traill}

The Food System: A Guide. By Geoff Tansey and Tony Worsley. Earthscan: 1995. Pp. 259. £15.95 (pbk).

PERHAPS it is unfair for an economist to review this book. Economists are mostly of the view that markets are a good thing and that the 'invisible hand' provides a cheap and efficient mechanism for allocating resources and rewarding effort. Of course, we recognize that markets are not perfect and that in certain cases political intervention is needed to regulate or otherwise remedy market failure: markets may become monopolized, 'externalities' may exist that are not taken into account by producers or consumers (such as the environmental implications of intensive farming or food packaging) and consumers may have inadequate knowledge to make informed choices (over food safety or nutritional issues, for example). The economist's response is to identify precisely the source of the market failure and take the necessary corrective action (provided that the benefits of doing so outweigh the costs).

The authors of this book start from the opposite position, believing that the market mechanism is fundamentally flawed and cannot be trusted to perform even the simplest tasks. They therefore conclude that the food system should be thoroughly managed to achieve a set of food-policy goals: the equitable provision of a safe, secure, sustainable, sufficient and nutritious diet for all. It is hard to argue with such basic objectives, the real question of course being whether politicians, civil servants and (much favoured by the authors) publicinterest nongovernmental organizations (PINGOs, as opposed to BINGOs, business and industry NGOs) can do a better job than a lightly regulated market of telling businesses what food to produce, and where and how, and consumers what to eat.

The authors spend most of the book describing their interpretation of the way in which the food system works in the developed world (with examples primarily from the United Kingdom and Australia) and where it is going wrong. A short set of conclusions discusses "general approaches" to the development of food policies for a new millennium.

In the book's favour, it does provide a lot of facts on a very wide range of subjects from ecology ("food and the biosphere") and food-poisoning organisms, through industrial and retailing change, to advertising and consumer market segments and much more besides. The book is divided into three main parts, the first dealing with global issues: it describes world agriculture (and its harmful effects on the environment); gives a short economic history of the world (and how it has resulted in a system in which richcountry citizens over-consume processed foods while poor-country citizens starve); and reviews nutrition science, food safety and the psychology and sociology of consumer food choice (and why we are all overweight and consequently exploited by clever advertising agents over our fears of not being slim and beautiful).

The second part of the book describes the principal actors in the food chain and their interaction. It covers farmers and the effects of agricultural policies on farming practices and farm income distribution, and the growing scale and global nature of the (often multinational) corporations involved in trading agricultural commodities, manufacturing food, sourcing produce and retailing and catering. It is not by this stage in the book a surprise to find that the authors believe that consumers have too little power in the system and that small-scale local operations are preferable to largescale global ones.

Power is returned to as the main theme in the final part of the book dealing with what the authors term "food control" mechanisms. These are the use of science and technology, information and sophisticated management techniques by actors to gain control in the food chain, and the use of policy to regulate the power and determine the distribution of benefits among the actors. There is the familiar argument that large corporations influence the allocation of research funds and so control the direction of technical progress. They also pay for advertising foods (and drinks) that are nutritionally harmful, and fund foodrelated (mis)education in schools. To top it all off, policies that could moderate these effects are formulated today by civil servants with male-centred ideologies and, according to an Australian survey quoted, a "pseudo-religious belief in the market".

Derek Cooper, a presenter of the British radio series "The Food Programme", is quoted on the front cover as saying: "if you want to understand why the world feeds itself the way it does, you must read this book". Be warned that, although one can have sympathy with many of the points the authors make, and despite an impression that the authors are often trying to appear impartial, the understanding one is fed is not entirely balanced.

Bruce Traill is in the Food Economics Group, University of Reading, Reading RG6 GAR, UK. 